GEF-TH-09/2006

\title{
General Solution Of Linear Vector Supersymmetry
}

\author{
Alberto Blasi and Nicola Maggiore 1 \\ Dipartimento di Fisica - Università di Genova - via Dodecaneso 33 - I-16146 Genova - \\ Italy and INFN, Sezione di Genova
}

\begin{abstract}
We give the general solution of the Ward identity for the linear vector supersymmetry which characterizes all topological models. Such solution, whose expression is quite compact and simple, greatly simplifies the study of theories displaying a supersymmetric algebraic structure, reducing to a few lines the proof of their possible finiteness. In particular, the cohomology technology usually involved for the quantum extension of these theories, is completely bypassed. The case of Chern-Simons theory is taken as an example.
\end{abstract}

Keywords: BRST Quantization, Chern-Simons Theories, Topological Field Theories, Supersymmetric Gauge Theories.

PACS Nos:

03.70.+k Theory of Quantized Fields;

11.15.-q Gauge Field Theories;

11.10.Gh Renormalization;

11.10.Nx Noncommutative Field Theory.

1ªlberto.blasi@ge.infn.ge, nicola.maggiore@ge.infn.it, 


\section{Introduction}

All topological field theories share that the energy-momentum tensor is not observable; for gauge field theories this feature is insured by a symmetry first discovered in [1]. We shall refer to it as the linear vector supersymmetry, which is encoded in a set $W_{\mu}$ of anticommuting Ward operators, carrying a spacetime index $\mu$.

Like the BRS symmetry, the form of the vector supersymmetry is the same for any field theory displaying it. The reason for this is its relation with the gauge fixing term, as discussed in [2], which, for any gauge field theory, is the same BRS cocycle. These two features of the vector supersymmetry, the fact of being shared by all topological field theories, and that of having the same functional form, render meaningful and interesting the task of finding its general solution.

In this paper we will show that, first, a general solution for the vector supersymmetry does exist, and it can be cast in a very compact form, and, second, that such solution is so constraining to be sufficient, alone, to completely determine the quantum extension of any topological field theory.

Usually, in the BRS approach to renormalization of gauge field theories, the attention is focused on the BRS identity, and the ensuing algebraic discussion of the cohomology spaces; the vector supersymmetry is then imposed on the solutions of the BRS cohomological problem. This constrains some otherwise free parameters and helps in proving the finiteness of the theory.

Here we would like to take the opposite attitude, and show that first discussing the general solution of the vector supersymmetry provides a more economical way towards the renormalization of topological gauge field theories and the discussion of observables in supersymmetric models.

Indeed, the general solution of the vector supersymmetry can be written in terms of $\mathcal{W} \equiv \varepsilon^{\mu_{1} \ldots \mu_{d}} W_{\mu_{1}} \ldots W_{\mu_{d}}$, so that in $d$ spacetime dimensions, the cohomological analysis of the linearized BRS operator is shifted from the sector with power counting dimension $p$ and ghost charge $q$, to the sector with power counting dimension $p-d$ and ghost charge $q+d$.

In all cases this results in a substantial simplification of the treatment.

For twisted models, where the vector supersymmetry does exist but it is not linearly realized, the question remains open; for instance, it is known that the Lagrangian of twisted $N=2$ Super Yang-Mills can be cast in this form, but this is not a general result.

In order not to overload the notation, we shall illustrate the general procedure of solving the vector supersymmetry Ward identities in the case of the ChernSimons theory with $d=3$, although the method can be straightforwardly extended to all other linear cases.

In section 2 we recall the defining functional equations for the Chern-Simons 
model, while the stability and anomaly problems are outlined in section 3 . In Section 4 we give the general solution for the vector supersymmetry Ward identity, which we use in Section 5 to show in a few lines the finiteness of the theory. Section 6 is devoted to our concluding remarks.

\section{The Classical Model}

Let us consider, as an example, the topological three dimensional ChernSimons (CS) theory, whose classical action is

$$
S_{C S}=\frac{k}{2} \int d^{3} x \epsilon^{\mu \nu \rho}\left(A_{\mu}^{a} \partial_{\nu} A_{\rho}^{a}+\frac{1}{3} f^{a b c} A_{\mu}^{a} A_{\nu}^{b} A_{\rho}^{c}\right),
$$

with the Landau gauge fixing

$$
S_{g f}=\int d^{3} x\left(b^{a} \partial A^{a}+\bar{c}^{a} \partial^{\mu}\left(D_{\mu} c\right)^{a}\right) .
$$

The fields $A_{\mu}^{a}(x), c^{a}(x), \bar{c}^{a}(x)$ and $b^{a}(x)$ represent gauge field, ghost, antighost and Lagrange multiplier (Nakanishi Lautrup field) respectively. They belong to the adjoint representation of a gauge group $G$, which we assume simple and compact. The $f^{a b c}$ are the structure constants of the Lie algebra, $k$ is the coupling constant and finally the adjoint covariant derivative is defined as

$$
\left(D_{\mu} c\right)^{a}=\partial_{\mu} c^{a}+f^{a b c} A_{\mu}^{b} c^{c} .
$$

Besides the usual, nilpotent, BRS symmetry

$$
\begin{aligned}
s A_{\mu}^{a} & =-\left(D_{\mu} c\right)^{a} \\
s c^{a} & =+\frac{1}{2} f^{a b c} c^{b} c^{c} \\
s \bar{c}^{a} & =b^{a} \\
s b^{a} & =0,
\end{aligned}
$$

under which $S_{C S}(A)$ and $S_{g f}(A, c, \bar{c}, b)$ are separately invariant

$$
s S_{C S}(A)=s S_{g f}(A, c, \bar{c}, b)=0,
$$

the gauge fixed action

$$
S=S_{C S}+S_{g f}
$$

shows an additional, vector supersymmetry [1]

$$
\delta_{\mu} S=0,
$$

where

$$
\begin{aligned}
\delta_{\mu} A_{\nu}^{a} & =\frac{1}{k} \epsilon_{\mu \nu \rho} \partial^{\rho} \bar{c}^{a} \\
\delta_{\mu} c^{a} & =-A_{\mu}^{a} \\
\delta_{\mu} \bar{c}^{a} & =0 \\
\delta_{\mu} b^{a} & =\partial_{\mu} \bar{c}^{a}
\end{aligned}
$$


whose origin is tightly related to the conservation of the energy-momentum tensor [2], which, for topological models, acquires contribution from the gauge fixing term only, since

$$
T_{\mu \nu}=\frac{\delta S}{\delta g^{\mu \nu}}=\frac{\delta S_{g f}}{\delta g^{\mu \nu}},
$$

where $g^{\mu \nu}$ is the metric describing the manifold on which the theory is built. Hence, the energy-momentum tensor is a BRS cocycle

$$
T_{\mu \nu}=s \Lambda_{\mu \nu} .
$$

The vector supersymmetry is not a feature of the particular model considered here, but its existence is a general property of all topological field theories, of both Witten and Schwartz type [4], built in the Landau gauge.

The set of constraints on the classical action $S$ is completed by the (Landau) gauge condition

$$
\frac{\delta S}{\delta b^{a}}=\partial A^{a}
$$

and the ghost condition

$$
\mathcal{G}^{a} S=\int d^{3} x\left(\frac{\delta}{\delta c^{a}}+f^{a b c} \bar{c}^{b} \frac{\delta}{\delta b^{c}}\right) S=0,
$$

which is peculiar to any gauge field theory in the Landau gauge [5].

The nonlinearity of the BRS transformations (2.4) renders necessary, in view of the quantum extension of the theory, the definition of the composite operators $s A_{\mu}^{a}$ and $s c^{a}$. This is done in a standard way in field theory, by coupling them to external sources (or antifields) $A^{\star a \mu}$ and $c^{\star a}$ by means of an additional term in the classical action :

$$
S_{\text {ext }}=\int d^{3} x\left(A^{\star a \mu} s A_{\mu}^{a}+c^{\star a} s c^{a}\right) .
$$

The complete classical action

$$
\Sigma=S_{C S}+S_{g f}+S_{e x t}
$$

satisfies

1. the Slavnov-Taylor (ST) identity

$$
\mathcal{S}(\Sigma)=\int d^{3} x\left(\frac{\delta \Sigma}{\delta A^{* a \mu}} \frac{\delta \Sigma}{\delta A_{\mu}^{a}}+\frac{\delta \Sigma}{\delta c^{a *}} \frac{\delta \Sigma}{\delta c^{a}}+b \frac{\delta \Sigma}{\delta \bar{c}}\right)=0,
$$

which is the functional transcription of the BRS symmetry (2.4);

2. the supersymmetric Ward identity assumes the same functional form in any topological field theory [4, 12.

$$
W_{\mu} \Sigma=\Delta_{\mu},
$$


where

$$
W_{\mu}=\int d^{3} x\left(\frac{1}{k} \epsilon_{\mu \nu \rho}\left(\partial^{\rho} \bar{c}^{a}+A^{a * \rho}\right) \frac{\delta}{\delta A_{\nu}^{a}}-A_{\mu}^{a} \frac{\delta}{\delta c^{a}}+\partial_{\mu} \bar{c}^{a} \frac{\delta}{\delta b^{a}}-c^{a *} \frac{\delta}{\delta A^{a * \mu}}\right)
$$

and $\Delta_{\mu}^{c l}$ is a breaking

$$
\Delta_{\mu}^{c l}=\int d^{3} x\left(\frac{1}{k} \epsilon_{\mu \nu \rho} A^{a * \nu} \partial^{\rho} b^{a}-A^{a * \nu} \partial_{\mu} A_{\nu}^{a}+c^{a *} \partial_{\mu} c^{a}\right) .
$$

As compared to (2.7), the vector supersymmetry on the complete classical action $\Sigma$, Eq. (2.16), appears to be a broken symmetry. But, since $\Delta_{\mu}^{c l}$ is only linear in the quantum fields, such a breaking concerns only the classical theory [6]. As we shall see, the Ward operator $W_{\mu}$ behaves as an exact symmetry for quantum objects such as counterterms and anomalies. This remark is crucial for what will follow;

3. the ghost equation, which, similarly to (2.16), on $\Sigma$ writes

$$
\mathcal{G}^{a} \Sigma=\Delta_{c l}^{a}
$$

where again $\Delta_{c l}^{a}$ is a classical breaking

$$
\Delta_{c l}^{a}=\int d^{3} x f^{a b c}\left(A^{b * \mu} A_{\mu}^{c}-c^{b *} c^{c}\right)
$$

4. the antighost condition

$$
\overline{\mathcal{G}}^{a} \Sigma=\left(\partial^{\mu} \frac{\delta}{\delta A^{a * \mu}}-\frac{\delta}{\delta \bar{c}^{a}}\right) \Sigma=0
$$

in virtue of which $A^{a * \mu}$ and $\bar{c}^{a}$ appear in the combination

$$
\widehat{A}_{\mu}^{\star a}=A_{\mu}^{a *}-\partial_{\mu} \bar{c}^{a}
$$

5. the Landau gauge condition (2.11), which formally remains the same

$$
\frac{\delta \Sigma}{\delta b^{a}}=\partial A^{a}
$$

The following nonlinear algebra, good for a generic functional $\gamma$, with even Faddeev-Popov charge, holds:

$$
\begin{aligned}
B_{\gamma} \mathcal{S}(\gamma) & =0 \\
\left\{W_{\mu}, W_{\nu}\right\} & =0 \\
W_{\mu} \mathcal{S}(\gamma)+B_{\gamma}\left(W_{\mu} \gamma-\Delta_{\mu}^{c l}\right) & =\mathcal{P}_{\mu} \gamma
\end{aligned}
$$


where $B_{\Sigma}$ is the linearized ST operator

$$
B_{\Sigma}=\int d^{3} x\left(\frac{\delta \Sigma}{\delta A^{* a \mu}} \frac{\delta}{\delta A_{\mu}^{a}}+\frac{\delta \Sigma}{\delta A_{\mu}^{a}} \frac{\delta}{\delta A^{a * \mu}}+\frac{\delta \Sigma}{\delta c^{a *}} \frac{\delta}{\delta c^{a}}+\frac{\delta \Sigma}{\delta c^{a}} \frac{\delta}{\delta c^{a *}}+b \frac{\delta \Sigma}{\delta \bar{c}}\right)=0
$$

and $\mathcal{P}_{\mu}$ is the translation Ward operator

$$
\mathcal{P}_{\mu}=\sum_{\text {all fields } \Phi} \int d^{3} x\left(\partial_{\mu} \Phi\right) \frac{\delta}{\delta \Phi} .
$$

If the functional $\gamma$ satisfies the ST identity (2.15) and the Ward identity (2.16), the algebra for the linear operator $B_{\Sigma}$ and $W_{\mu}$ is

$$
\begin{aligned}
\left(B_{\Sigma}\right)^{2} & =0 \\
\left\{W_{\mu}, W_{\nu}\right\} & =0 \\
\left\{B_{\Sigma}, W_{\mu}\right\} & =\mathcal{P}_{\mu} .
\end{aligned}
$$

Notice that the introduction of the external sources $A^{\star a \mu}$ and $c^{\star a}$ allows to put off shell the algebra [1]. Under this respect, the external sources, which naturally enter the game in the BRS formalism, play exactly the role of the auxiliary fields of the ordinary supersymmetry [7].

\section{The Quantum Theory}

The quantum theory is well defined once we have proved that the divergences can be reabsorbed through a redefinition of fields and parameters of the theory, order by order in perturbation theory, and once we have been able to show the absence of quantum obstructions for the symmetries defining the theory.

In other words, the quantum extension of a generic field theory goes through the determination of the counterterm and of the proof of the absence of anomalies.

Topological fields theories, like the one we are considering in this paper, are an example of finite field theories [2]. This means that no counterterm should be allowed, i.e. a finite model does not display any divergence which needs to be reabsorbed.

As far as we know, the only field theories showing this remarkable property are topological models and supersymmetric gauge field theories [7]. It is not by chance, indeed, that topological fields theories are characterized, through the presence of the linear vector supersymmetry $W_{\mu}$, by a typically supersymmetric algebra like (2.29) [2, 8, 9, 10, 11, 12]. In some cases, ordinary gauge field theories and topological quantum field theories even coincide, being possible to pass from one to the other by means of a twisting procedure [13]. This is the case, for instance, of $\mathrm{N}=2$ Super Yang Mills (SYM) 
theory and topological Yang-Mills (TYM) theory [14, 15], where the vector supersymmetry is not linearly realized, and our method cannot be applied straightforwardly.

For CS theory, finiteness has been proved in [16] and in [17], independently, and following two different approaches. In this paper we give an alternative proof, and, even if we believe that the method presented here gives a new, particularly quick and economic demonstration of the finiteness of the theory, we stress again that our main aim is to point out the power of supersymmetry, and of its general solution which will be given in the next section. Supersymmetry alone turns out to be sufficient to completely determine the quantum extension of these theories.

A generic local integrated functional $X_{q}^{p}$ of power counting dimension $p$ and ghost number $q$ which satisfies the algebraic constraints beyond the classical level obeys

$$
\begin{aligned}
\frac{\delta X_{q}^{p}}{\delta b^{a}(x)} & =0 \\
\overline{\mathcal{G}}^{a}(x) X_{q}^{p} & =0 \\
\mathcal{G}^{a} X_{q}^{p} & =0 \\
W_{\mu} X_{q}^{p} & =0 \\
B_{\Sigma} X_{q}^{p} & =0 .
\end{aligned}
$$

It is well known [6] that the linear symmetries (3.1), (3.2) and (3.3) can be extended to the quantum level, and therefore we have to analyze (3.4) and (3.5), the relevant cases for the quantum extension of the theory being $X_{0}^{3}$ for the counterterm and $X_{1}^{3}$ for the anomaly.

\section{General Solution Of Supersymmetry Constraint}

The aim of this section, is to study the most general solution of the supersymmetry constraint (3.4)

$$
W_{\mu} X_{q}^{p}=0 .
$$

The interest for solving this equation is evident, since it must be satisfied by both counterterm and gauge anomaly, in topological and (twisted) supersymmetric field theories as well. This, together with the fact that the form of $W_{\mu}$ is independent from the particular model considered, being related only to the gauge fixing term, according to (2.9), justifies our effort for finding its general solution.

In the functional space defined by the gauge condition (3.1), the antighost equation (3.2) and the ghost equation (3.3), the Ward operator $W_{\mu}$ reads

$$
W_{\mu}=\int d^{3} x\left(\frac{1}{k} \varepsilon_{\mu \nu \rho} \hat{A}^{a * \rho} \frac{\delta}{\delta A_{\nu}^{a}}-A_{\mu}^{a} \frac{\delta}{\delta c^{a}}+c^{a *} \frac{\delta}{\delta \hat{A}^{a * \mu}}\right),
$$


and it satisfies the algebraic relation (2.25).

It is convenient to make the Lorentz index explicit:

$$
\begin{aligned}
& W_{1}=\int d^{3} x\left(\frac{1}{k} \hat{A}_{3}^{a *} \frac{\delta}{\delta A_{2}^{a}}-\frac{1}{k} \hat{A}_{2}^{a *} \frac{\delta}{\delta A_{3}^{a}}-A_{1}^{a} \frac{\delta}{\delta c^{a}}+c^{a *} \frac{\delta}{\delta \hat{A}_{1}^{a *}}\right) \\
& W_{2}=\int d^{3} x\left(-\frac{1}{k} \hat{A}_{3}^{a *} \frac{\delta}{\delta A_{1}^{a}}+\frac{1}{k} \hat{A}_{1}^{a *} \frac{\delta}{\delta A_{3}^{a}}-A_{2}^{a} \frac{\delta}{\delta c^{a}}+c^{a *} \frac{\delta}{\delta \hat{A}_{2}^{a *}}\right) \\
& W_{3}=\int d^{3} x\left(\frac{1}{k} \hat{A}_{2}^{a *} \frac{\delta}{\delta A_{1}^{a}}-\frac{1}{k} \hat{A}_{1}^{a *} \frac{\delta}{\delta A_{2}^{a}}-A_{3}^{a} \frac{\delta}{\delta c^{a}}+c^{a *} \frac{\delta}{\delta \hat{A}_{3}^{a *}}\right)
\end{aligned}
$$

The algebra between the $W$ 's is

$$
\begin{gathered}
W_{1}^{2}=W_{2}^{2}=W_{3}^{2}=0 \\
\left\{W_{1}, W_{2}\right\}=\left\{W_{1}, W_{3}\right\}=\left\{W_{2}, W_{3}\right\}=0 .
\end{gathered}
$$

In particular, the three operators $W$ are nilpotent. Now, from their explicit expressions, we see that all fields forming the functional space on which the $W$ 's act, appear as BRS doublets [6], i.e. are of the type:

$$
W_{i} \phi=\psi \quad, \quad W_{i} \psi=0 \quad i=1,2,3
$$

or, equivalently

$$
\left\{W_{i}, W_{j}^{\dagger}\right\}=\delta_{i j} \mathcal{N},
$$

where the $W_{i}^{\dagger}$ are the functional adjoints of $W_{i}$

$$
\begin{aligned}
W_{1}^{\dagger} & =\int d^{3} x\left(k A_{2}^{a} \frac{\delta}{\delta \hat{A}_{3}^{a *}}-k A_{3}^{a} \frac{\delta}{\delta \hat{A}_{2}^{a *}}-c^{a} \frac{\delta}{\delta A_{1}^{a}}+\hat{A}_{1}^{a *} \frac{\delta}{\delta c^{a *}}\right) \\
W_{2}^{\dagger} & =\int d^{3} x\left(-k A_{1}^{a} \frac{\delta}{\delta \hat{A}_{3}^{a *}}+k A_{3}^{a} \frac{\delta}{\delta \hat{A}_{1}^{a *}}-c^{a} \frac{\delta}{\delta A_{2}^{a}}+\hat{A}_{2}^{a *} \frac{\delta}{\delta c^{a *}}\right) \\
W_{3}^{\dagger} & =\int d^{3} x\left(k A_{1}^{a} \frac{\delta}{\delta \hat{A}_{2}^{a *}}-k A_{2}^{a} \frac{\delta}{\delta \hat{A}_{1}^{a *}}-c^{a} \frac{\delta}{\delta A_{3}^{a}}+\hat{A}_{3}^{a *} \frac{\delta}{\delta c^{a *}}\right)
\end{aligned}
$$

and the operator $\mathcal{N}$

$$
\mathcal{N}=\int d^{3} x \sum_{\text {all fields } \Phi} \Phi \frac{\delta}{\delta \Phi}
$$

counts the number $n_{\Phi}$ of fields $\Phi$ appearing in a generic functional $\mathcal{F}(\Phi)$

$$
\mathcal{N} \mathcal{F}(\Phi)=\sum_{\text {all fields } \Phi} n_{\Phi} \mathcal{F}(\Phi) \equiv N \mathcal{F}(\Phi)
$$

According to a well known theorem concerning nilpotent operators [6], fields transforming as BRS doublets, do not belong to the cohomology. This means 
that the cohomology of each $W_{i}$ is empty, since all fields are organized in BRS doublets, and that the solutions of the equations

$$
W_{i} X_{q}^{p}=0
$$

can be written as

$$
X_{q}^{p}=W_{i} X_{q+1}^{p-1},
$$

where we took into account the fact that the operators $W$ raise by one unit the mass dimensions and lower the ghost number by the same amount.

But we can go further. We are looking, indeed, for the most general $X_{q}^{p}$ which satisfies the set of equations

$$
W_{1} X_{q}^{p}=W_{2} X_{q}^{p}=W_{3} X_{q}^{p}=0 .
$$

Since the cohomology of each $W_{i}$ is empty, we have

$$
X_{q}^{p}=W_{1} \bar{X}_{q+1}^{p-1}=W_{2} \widehat{X}_{q+1}^{p-1}=W_{3} \widetilde{X}_{q+1}^{p-1},
$$

where $\bar{X}_{q+1}^{p-1}, \widehat{X}_{q+1}^{p-1}$ and $\widetilde{X}_{q+1}^{p-1}$ are generic functionals.

Multiplying both sides of the second identity in (4.18) by $W_{1}^{\dagger}$, we have

$$
W_{1}^{\dagger} W_{1} \bar{X}_{q+1}^{p-1}=W_{1}^{\dagger} W_{2} \widehat{X}_{q+1}^{p-1},
$$

and, using (4.9), we get

$$
\mathcal{N} \bar{X}_{q+1}^{p-1}-W_{1} W_{1}^{\dagger} \bar{X}_{q+1}^{p-1}=-W_{2} W_{1}^{\dagger} \widehat{X}_{q+1}^{p-1} .
$$

Thanks to (4.14), we can write

$$
\bar{X}_{q+1}^{p-1}=\frac{1}{N_{1}}\left(W_{1} W_{1}^{\dagger} \bar{X}_{q+1}^{p-1}-W_{2} W_{1}^{\dagger} \widehat{X}_{q+1}^{p-1}\right),
$$

where $N_{1}$ is the number of fields appearing in $\bar{X}_{q+1}^{p-1}$. Similarly, from the third identity in (4.18), we have

$$
W_{2}^{\dagger} W_{2} \widehat{X}_{q+1}^{p-1}=W_{2}^{\dagger} W_{3} \widetilde{X}_{q+1}^{p-1},
$$

hence

$$
\mathcal{N} \widehat{X}_{q+1}^{p-1}-W_{2} W_{2}^{\dagger} \widehat{X}_{q+1}^{p-1}=-W_{3} W_{2}^{\dagger} \widetilde{X}_{q+1}^{p-1}
$$

i.e.

$$
\widehat{X}_{q+1}^{p-1}=\frac{1}{N_{2}}\left(W_{2} W_{2}^{\dagger} \widehat{X}_{q+1}^{p-1}-W_{3} W_{2}^{\dagger} \widetilde{X}_{q+1}^{p-1}\right),
$$

where $N_{2}$ is the number of fields appearing in $\widehat{X}_{q+1}^{p-1}$.

Substituting (4.24) in (4.21), we get

$$
\begin{aligned}
\bar{X}_{q+1}^{p-1} & =\frac{1}{N_{1}} W_{1} W_{1}^{\dagger} \bar{X}_{q+1}^{p-1}-\frac{1}{N_{1} N_{2}} W_{2} W_{1}^{\dagger}\left(W_{2} W_{2}^{\dagger} \widehat{X}_{q+1}^{p-1}-W_{3} W_{2}^{\dagger} \widetilde{X}_{q+1}^{p-1}\right) \\
& =\frac{1}{N_{1}} W_{1} W_{1}^{\dagger} \bar{X}_{q+1}^{p-1}+\frac{1}{N_{1} N_{2}} W_{2} W_{1}^{\dagger} W_{3} W_{2}^{\dagger} \widetilde{X}_{q+1}^{p-1}
\end{aligned}
$$


because of the nilpotency of $W_{2}$ and of the algebra (4.9).

We are now able to write, from (4.18)

$$
X_{q}^{p}=\frac{1}{N_{1} N_{2}} W_{1} W_{2} W_{1}^{\dagger} W_{3} W_{2}^{\dagger} \widetilde{X}_{q+1}^{p-1}
$$

Therefore, the most general solution of the equations (4.17) is, by construction

$$
X_{q}^{p}=W_{1} W_{2} W_{3} Y_{q+3}^{p-3} .
$$

In covariant notations our result writes

$$
W_{\mu} X_{q}^{p}=0 \rightleftharpoons X_{q}^{p}=\varepsilon^{\alpha \beta \gamma} W_{\alpha} W_{\beta} W_{\gamma} Y_{q+3}^{p-3}
$$

where $Y_{q+3}^{p-3}$ is a generic functional with dimensions $p-3$ and ghost number $q+3$.

Although our proof has been given in three dimensions, the procedure we employed holds for any topological field theory in $d$-spacetime, and hence we can write the most general solution of the set of $d$ equations

$$
W_{\mu} X_{q}^{p}=0
$$

as

$$
X_{q}^{p}=\mathcal{W} X_{q+d}^{p-d}
$$

where

$$
\mathcal{W} \equiv \varepsilon^{\mu_{1} \ldots \mu_{d}} W_{\mu_{1}} \ldots W_{\mu_{d}} .
$$

The fact that the operators $W_{\mu}$ carry both dimensions and Faddeev-Popov charge, is quite constraining, and the consequences on counterterm and anomalies are spectacular, as we shall see in the next section.

We finally stress that, due to the algebra (2.25), a functional written as (4.30) trivially satisfies the supersymmetry equation (4.29). What we have shown here, is that no other solutions exist.

\section{$5 \quad$ Supersymmetry And Finiteness}

The cohomology equations (3.5), written for the counterterm and for the anomaly, are usually considered, and generally are, as the hearth of the quantum determination of the theory, and of the algebraic renormalization.

Smart and subtle techniques have been developed for solving them, like the method of spectral sequences [24] or the so called "russian formula" 25], and useful theorems exist, which give information on their solutions [6]. Brute force, on the other hand, often required a lot of time and hard work to obtain important results concerning the renormalization (or non renormalization) of the theory. What we want to show in this paper, is that for the large number 
of theories which exhibit the vector supersymmetry, the way to gain quantum theory is much shorter and bypasses the cohomology technology.

Let us begin with the counterterm $X_{0}^{3}$, which must satisfy the supersymmetry condition (3.4).

We have just shown that the most general solution is

$$
X_{0}^{3}=\mathcal{W} X_{3}^{0},
$$

where $X_{3}^{0}$ is an integrated local functional with vanishing power counting dimension and ghost number three. The only possibility is

$$
X_{3}^{0}=\alpha \int d^{3} x f^{a b c} c^{a} c^{b} c^{c},
$$

where $\alpha$ is a coefficient. This only candidate for the counterterm is ruled out by the ghost condition (3.3), because of the algebraic condition

$$
\left\{\mathcal{G}^{a}, \mathcal{W}\right\}=0
$$

which can be easily verified.

Hence,

$$
X_{0}^{3}=0,
$$

which proves the finiteness of the theory.

The proof of absence of anomalies is even shorter. Besides the Wess-Zumino consistency condition (3.5), the gauge anomaly $X_{1}^{3}$ must satisfy the supersymmetry equation (3.4), whose most general solution is

$$
X_{1}^{3}=\mathcal{W} X_{4}^{0}
$$

where $X_{4}^{0}$ is a generic integrated local functional functional with mass dimension zero and Faddeev-Popov charge four.

The only possibility would be

$$
X_{4}^{0}=\int d^{3} x T^{a b c d} c^{a} c^{b} c^{c} c^{d}
$$

but such candidate is not there, simply because an invariant tensor $T^{a b c d}$, which must be completely antisymmetric in its four color indices, cannot even be written [26].

That's the reason why

$$
X_{1}^{3}=0,
$$

and therefore no anomalies exist for any of the symmetries defining the model. Notice that, to show the results (5.4) and (5.7), no use has been done of the left conditions on the counterterm and on the anomaly, which are by far the toughest ones to analyze, i.e. the ST conditions (3.5). As promised. 


\section{An Example: Absence Of Anomalies In Noncommu- tative Chern-Simons Theory}

Besides finiteness of Chern-Simons theory, as another, original, application of our result (4.30), in this section we face the problem of anomalies for noncommutative Chern-Simons (NCCS) theory.

As it is well known, a noncommutative extension of a generic quantum field theory is obtained through the substitution of the ordinary product with the Groenewald-Moyal [27, 28]:

$$
\phi(x) \psi(x) \longrightarrow \phi(x) * \psi(x) \equiv \lim _{y \rightarrow x} \exp \left(\frac{i}{2} \theta^{\mu \nu} \partial_{\mu}^{x} \partial_{\nu}^{y}\right) \phi(x) \psi(y)
$$

where $\theta^{\mu \nu}$ is a rank-two antisymmetric matrix which controls the noncommutative nature of spacetime coordinates

$$
\left[x^{\mu}, x^{\nu}\right]=i \theta^{\mu \nu}
$$

The NCCS theory, at $O\left(\theta^{2}\right)$, reads

$$
\begin{aligned}
S_{N C C S}= & \frac{k}{2} \operatorname{Tr} \int d^{3} x \epsilon^{\mu \nu \rho}\left(A_{\mu} * \partial_{\nu} A_{\rho}-i \frac{2}{3} A_{\mu} * A_{\nu} * A_{\rho}\right) \\
= & S_{C S}+\theta^{\alpha \beta} \int d^{3} x\left(\frac{1}{12} \varepsilon^{\mu \nu \rho} d^{a b c}\left(\partial_{\alpha} A_{\mu}^{a}\right)\left(\partial_{\beta} A_{\nu}^{b}\right) A_{\rho}^{c}\right)+ \\
& +\theta^{\alpha \beta} \theta^{\gamma \delta} \int d^{3} x\left(-\frac{1}{48} \varepsilon^{\mu \nu \rho} f^{a b c}\left(\partial_{\alpha \gamma} A_{\mu}^{a}\right)\left(\partial_{\beta \delta} A_{\nu}^{b}\right) A_{\rho}^{c}\right) .
\end{aligned}
$$

The noncommutative gauge fixing term, , at $O\left(\theta^{2}\right)$, is

$$
\begin{aligned}
S_{N C g f}= & s^{(\theta)} \operatorname{Tr} \int d^{3} x \bar{c} * \partial^{\mu} A_{\mu} \\
= & S_{g f}+\theta^{\alpha \beta} \int d^{3} x\left(-\frac{1}{2} d^{a b c}\left(\partial^{\mu} \bar{c}^{a}\right)\left(\partial_{\alpha} A_{\mu}^{b}\right)\left(\partial_{\beta} c^{c}\right)\right)+ \\
& +\theta^{\alpha \beta} \theta^{\gamma \delta} \int d^{3} x\left(\frac{1}{8} f^{a b c} \partial^{\mu} \bar{c}^{a}\left(\partial_{\alpha \gamma} A_{\mu}^{b}\right)\left(\partial_{\beta \delta} c^{c}\right)\right)
\end{aligned}
$$

where $s^{(\theta)}$ is the noncommutative extension of the ordinary BRS operator (2.4), always at $O\left(\theta^{2}\right)$ :

$$
\begin{aligned}
s^{(\theta)} A_{\mu}^{a} & =s A_{\mu}^{a}-\frac{1}{2} \theta^{\alpha \beta} d^{a b c} \partial_{\alpha} A_{\mu}^{b} \partial_{\beta} c^{c}+\frac{1}{8} \theta^{\alpha \beta} \theta^{\gamma \delta} f^{a b c} \partial_{\alpha \gamma} A_{\mu}^{b} \partial_{\beta \delta} c^{c} \\
s^{(\theta)} c^{a} & =s c^{a}+\frac{1}{4} \theta^{\alpha \beta} d^{a b c} \partial_{\alpha} c^{b} \partial_{\beta} c^{c}-\frac{1}{16} \theta^{\alpha \beta} \theta^{\gamma \delta} f^{a b c} \partial_{\alpha \gamma} c^{b} \partial_{\beta \delta} c^{c} \\
s^{(\theta)} \bar{c}^{a} & =s \bar{c}^{a} \\
s^{(\theta)} b^{a} & =s b^{a} .
\end{aligned}
$$


The noncommutative action (6.3), although quite different from the ordinary one (2.1), is still invariant under supersymmetry, which, being a linear transformation, is not modified by $\theta$ :

$$
\delta_{\mu}\left(S_{N C C S}+S_{N C g f}\right)=0 .
$$

The introduction of the noncommutative parameter deeply alters the nature of any theory, firstly because it breaks Lorentz invariance. In addition, it has been shown that, at least in the two-dimensional noncommutative BF model [29, 30], the $\theta$-deformed theory is not stable under radiative corrections, since the presence of $\theta$ opens a sector for counterterms which are not present at the classical level, and which cannot be traced back to a Groenewald-Moyal product, which turns out to be unstable as well.

As an open question remains the issue of anomalies, since there are no results concerning the cohomology of the BRS operator in presence of a $\theta$-parameter 31. The fact that $\theta$ has negative mass dimensions, renders the algebraic analysis overwhelmingly heavy, and, in practice, results at higher orders in $\theta$ turn out to be out of reach.

Our result considerably simplifies the analysis, and the algebraic treatment, even at higher orders in $\theta$, is still feasible.

The 3D gauge anomaly is a local integrated functional $\mathcal{A}_{1}^{(\theta) 3}$ with mass dimensions +3 and ghost charge +1 , which satisfies all the symmetries defining the classical action, namely the ghost equation

$$
\mathcal{G}^{a} \mathcal{A}_{1}^{(\theta) 3}=0
$$

the supersymmetry condition

$$
W_{\mu} \mathcal{A}_{1}^{(\theta) 3}=0
$$

and, finally, the Slavnov-Taylor condition

$$
B_{\Sigma}^{(\theta)} \mathcal{A}_{1}^{(\theta) 3}=0
$$

Concerning the last constraint, namely the Wess-Zumino condition, in order that the functional $\mathcal{A}_{1}^{(\theta) 3}$ is a true anomaly, it must belong to the cohomology of $B_{\Sigma}^{(\theta)}$, namely:

$$
\mathcal{A}_{1}^{(\theta) 3} \neq B_{\Sigma}^{(\theta)} \mathcal{A}_{0}^{(\theta) 3}
$$

In the noncommutative case, $\mathcal{A}_{1}^{(\theta) 3}$ can be expressed as a power series in 61 :

$$
\mathcal{A}_{1}^{(\theta) 3}=\mathcal{A}_{1}^{3}+\sum_{n=1}^{\infty} \theta_{\alpha_{1} \beta_{1} . .} \theta_{\alpha_{n} \beta_{n}}\left(\mathcal{A}_{1}^{3+2 n}\right)^{\alpha_{1} \beta_{1} . . \alpha_{n} \beta_{n}} .
$$

\footnotetext{
${ }^{1}$ It can be easily proved that in $3 \mathrm{D}$ no non-analytical sector exists in $\theta$.
} 
Since the linear operator $W_{\mu}$ is $\theta$-independent, the equation (6.8) must hold order by order in $\theta$ :

$$
W_{\mu}\left(\mathcal{A}_{1}^{3+2 n}\right)^{\alpha_{1} \beta_{1} . . \alpha_{n} \beta_{n}}=0,
$$

which is the equation whose general solution we give in this paper, according to which

$$
\left(\mathcal{A}_{1}^{3+2 n}\right)^{\alpha_{1} \beta_{1} . . \alpha_{n} \beta_{n}}=\mathcal{W}\left(\mathcal{A}_{4}^{2 n}\right)^{\alpha_{1} \beta_{1} . . \alpha_{n} \beta_{n}},
$$

i.e., to find candidates for the anomaly at the order $\theta^{n}$, we must study the cohomology of the Slavnov-Taylor operator in the space of local integrated functionals with mass dimension $2 n$, ghost charge +4 , with $2 n$ Lorentz indices. This greatly reduces the number of possibilities.

Indeed:

The (commutative) order $\theta^{0}$ has already been treated in the previous section, with the immediate outcome that no commutative anomaly exists.

At order $O(\theta)$ :

$$
\left(\mathcal{A}_{1}^{5}\right)^{\alpha \beta}=\mathcal{W}\left(\mathcal{A}_{4}^{2}\right)^{\alpha \beta},
$$

and we can immediately conclude that no anomalies exist at this order, since no functional $\left(\mathcal{A}_{4}^{2}\right)^{\alpha \beta}$, with mass dimensions +2 and ghost charge +4 can be written, which satisfies the ghost equation (6.7).

At order $O\left(\theta^{2}\right)$ :

$$
\left(\mathcal{A}_{1}^{7}\right)^{\alpha_{1} \beta_{1} \alpha_{2} \beta_{2}}=\mathcal{W}\left(\mathcal{A}_{4}^{4}\right)^{\alpha_{1} \beta_{1} \alpha_{2} \beta_{2}} .
$$

There are three possibilities:

$$
\begin{aligned}
& \left(\mathcal{A}_{4}^{4}\right)_{(1)}^{\alpha_{1} \beta_{1} \alpha_{2} \beta_{2}}=\delta^{\alpha_{1} \alpha_{2}} \delta^{\beta_{1} \beta_{2}} T_{1}^{a b c d} \int d^{3} x\left(\partial^{\mu} c^{a}\right)\left(\partial_{\mu} c^{b}\right)\left(\partial^{\nu} c^{c}\right)\left(\partial_{\nu} c^{d}\right)(6.1 \\
& \left(\mathcal{A}_{4}^{4}\right)_{(2)}^{\alpha_{1} \beta_{1} \alpha_{2} \beta_{2}}=\delta^{\alpha_{1} \alpha_{2}} T_{2}^{a b c d} \int d^{3} x\left(\partial^{\beta_{1}} c^{a}\right)\left(\partial^{\beta_{2}} c^{b}\right)\left(\partial^{\mu} c^{c}\right)\left(\partial_{\mu} c^{d}\right) \\
& \left(\mathcal{A}_{4}^{4}\right)_{(3)}^{\alpha_{1} \beta_{1} \alpha_{2} \beta_{2}}=T_{3}^{a b c d} \int d^{3} x\left(\partial^{\alpha_{1}} c^{a}\right)\left(\partial^{\alpha_{2}} c^{b}\right)\left(\partial^{\beta_{1}} c^{c}\right)\left(\partial^{\beta_{2}} c^{d}\right)
\end{aligned}
$$

where $T_{i}^{a b c d}$ are invariant tensors.

Due to the algebraic relation

$$
\left\{B_{\Sigma}, \mathcal{W}\right\}=0
$$

which holds in the space of integrated functionals, the anomaly candidates are $B_{\Sigma^{-}}$-invariant. Nevertheless, it is immediate to recognize that, using again (6.19), none of the candidates belong to the BRS cohomology, therefore, no $\theta$-dependent anomaly exists, at least at order $O\left(\theta^{2}\right)$.

We could easily carry on the analysis at higher orders in $\theta$, to finally prove that no $\theta$-dependent anomaly exists at all, thus leading to the nontrivial result that the noncommutative extension does not introduce anomalies, at least in Chern-Simons theory. But the aim of this section is just to give an example of the power of the result (4.30), namely the most general solution of the supersymmetry equation, which allows a much easier analysis of issues otherwise technically quite involved. 


\section{Conclusions}

We have shown, on the example of the Chern-Simons model in three spacetime dimensions, that tackling the problem of its renormalizability by first solving for the linear vector supersymmetry, considerably shortens the algebraic work to be done; in particular, the absence of counterterms (finiteness) and of anomalies reduces just to a couple of lines.

The reason for this, is that the most general solution of the linear vector supersymmetry has the form (4.30), expressed by means of the operator $\mathcal{W}$ (4.31), which shifts the problem to a much smaller functional space, being characterized by a higher ghost charge, and zero dimensionality.

We remark that, this property is related to the functional form of the operator $W_{\mu}$, which is the same for any topological field theory, since it depends only on the gauge fixing part of the action and not on the particular model considered.

It would be nice if this result could be extended to twisted topological field theories; here we have to face the problem that the vector supersymmetry is not linearly realized, and therefore the study of the cohomology of the $W_{\mu}$ operators is not straightforward. In particular, for the twisted $N=2 \mathrm{SYM}$ model, it is known that the Lagrangian can be written as

$$
\Sigma_{N=2 S Y M}=\mathcal{W} X
$$

but only modulo an exact BRS cocycle, and there are $W_{\mu}$ invariant fields which suggest that the cohomology of the $W_{\mu}$ operators might not be empty. Further investigations towards the generalization of our result to theories with nonlinear twisted supersymmetry are in progress.

What we have shown in this paper, is that any theory displaying a linear vector supersymmetry can be put in the form (7.1), and hence shows relevant nonrenormalization properties.

Another point that we would like to stress, is that in the discussion of the renormalization of these supersymmetric models, it has not been necessary to solve any cohomological BRS condition, contrarily to what happens in ordinary cases. This is the simplification we mentioned more than once in this paper. Curiously enough, we here have a class of gauge field theories whose quantum extension is not determined by any BRS constraint. Nevertheless the gauge field theory nature of these models is apparent, being deeply encoded in the set of non gauge symmetries which are involved. We remind, indeed, that both the ghost equation (2.12) and the supersymmetry (2.16) hold only if the Landau gauge is adopted. Moreover, for the classical action both the vector supersymmetry and the ghost equation are broken, so its determination is most easily and naturally done in terms of the standard BRS approach.

The hope is that a similar treatment, applied to noncommutative topological field theory models [27, 28, will help in the algebraic analysis of stability 
and anomaly. Indeed, the presence of the $\theta_{\mu \nu}$ parameter with negative power counting dimensions, raises very rapidly the dimensions of the field dependent breakings. Therefore, the possibility of lowering it by means of the solution of the vector supersymmetry looks like a promising way of attacking the problem. Work is in progress in this direction.

\section{References}

[1] F. Delduc, F. Gieres and S. P. Sorella, Supersymmetry Of The $D=3$ Chern-Simons Action In The Landau Gauge, Phys. Lett. B 225, 367 (1989).

[2] N. Maggiore and S. P. Sorella, Finiteness of the topological models in the Landau gauge, Nucl. Phys. B 377, 236 (1992).

[3] L. Alvarez-Gaume, J. M. F. Labastida and A. V. Ramallo, A Note On Perturbative Chern-Simons Theory, Nucl. Phys. B 334, 103 (1990).

[4] D. Birmingham, M. Blau, M. Rakowski and G. Thompson, Topological field theory, Phys. Rept. 209, 129 (1991).

[5] A. Blasi, O. Piguet and S. P. Sorella, Landau Gauge And Finiteness, Nucl. Phys. B 356, 154 (1991).

[6] O. Piguet and S. P. Sorella, Algebraic renormalization: Perturbative renormalization, symmetries and anomalies, Lect. Notes Phys. M28, 1 (1995).

[7] P. C. West, Introduction To Supersymmetry And Supergravity, World Scientific (1990), Singapore.

[8] E. Guadagnini, N. Maggiore and S. P. Sorella, Supersymmetry Of The Three-Dimensional Einstein-Hilbert Gravity In The Landau Gauge, Phys. Lett. B 247, 543 (1990).

[9] E. Guadagnini, N. Maggiore and S. P. Sorella, Supersymmetric Structure Of Four-Dimensional Antisymmetric Tensor Fields, Phys. Lett. B 255, 65 (1991).

[10] N. Maggiore and S. P. Sorella, Perturbation theory for antisymmetric tensor fields in four-dimensions, Int. J. Mod. Phys. A 8, 929 (1993) arXiv:hep-th/9204044.

[11] A. Blasi and N. Maggiore, Infrared and ultraviolet finiteness of topological BF theory in two-dimensions, Class. Quant. Grav. 10, 37 (1993) arXiv:hep-th/9207008. 
[12] D. Birmingham and M. Rakowski, Vector supersymmetry in topological field theory, Phys. Lett. B 269, 103 (1991).

[13] E. Witten, Topological Quantum Field Theory, Commun. Math. Phys. 117, 353 (1988).

[14] F. Fucito, A. Tanzini, L. C. Q. Vilar, O. S. Ventura, C. A. G. Sasaki and S. P. Sorella, Algebraic renormalization: Perturbative twisted considerations on topological Yang-Mills theory and on $N=2$ supersymmetric gauge theories, arXiv:hep-th/9707209.

[15] A. Blasi, V. E. R. Lemes, N. Maggiore, S. P. Sorella, A. Tanzini, O. S. Ventura and L. C. Q. Vilar, Perturbative beta function of $N=2$ super Yang-Mills theories, JHEP 0005, 039 (2000) arXiv:hep-th/0004048.

[16] A. Blasi and R. Collina, Finiteness Of The Chern-Simons Model In Perturbation Theory, Nucl. Phys. B 345, 472 (1990).

[17] F. Delduc, C. Lucchesi, O. Piguet and S. P. Sorella, Exact Scale Invariance Of The Chern-Simons Theory In The Landau Gauge, Nucl. Phys. B 346, 313 (1990).

[18] J. H. Lowenstein, Normal Product Quantization Of Currents In Lagrangian Field Theory, Phys. Rev. D 4, 2281 (1971).

[19] J. H. Lowenstein, Differential Vertex Operations In Lagrangian Field Theory, Commun. Math. Phys. 24, 1 (1971).

[20] Y. M. Lam, Perturbation Lagrangian Theory For Scalar Fields: WardTakahasi Identity And Current Algebra, Phys. Rev. D 6, 2145 (1972).

[21] Y. M. Lam, Equivalence Theorem On Bogolyubov-Parasiuk-HeppZimmermann Renormalized Lagrangian Field Theories, Phys. Rev. D 7, 2943 (1973).

[22] T. E. Clark and J. H. Lowenstein, Generalization Of Zimmermann's Normal - Product Identity, Nucl. Phys. B 113, 109 (1976).

[23] J. Wess and B. Zumino, Consequences Of Anomalous Ward Identities, Phys. Lett. B 37, 95 (1971).

[24] J. A. Dixon, Calculation of BRS cohomology with spectral sequences, Commun. Math. Phys. 139, 495 (1991).

[25] B. Zumino, Chiral Anomalies And Differential Geometry: Lectures Given At Les Houches, August 1983. 
[26] A. J. MacFarlane, A. Sudbery and P. H. Weisz, On GellMann's Gamma Matrices, D Tensors And F Tensors, Octets, And Parametrizations Of SU(3), Commun. Math. Phys. 11, 77 (1968).

[27] M. R. Douglas and N. A. Nekrasov, Noncommutative field theory, Rev. Mod. Phys. 73, 977 (2001) [arXiv:hep-th/0106048].

[28] R. J. Szabo, Quantum field theory on noncommutative spaces, Phys. Rept. 378, 207 (2003) arXiv:hep-th/0109162.

[29] A. Blasi, N. Maggiore and M. Montobbio, Instabilities of noncommutative two dimensional BF model, Mod. Phys. Lett. A 20, 2119 (2005) arXiv:hep-th/0504218.

[30] A. Blasi, N. Maggiore and M. Montobbio, Noncommutative two dimensional BF model, Nucl. Phys. B 740, 281 (2006) arXiv:hep-th/0512006].

[31] M. Picariello, A. Quadri and S. P. Sorella, Chern-Simons in the SeibergWitten map for non-commutative Abelian gauge theories in $4 D$, JHEP 0201, 045 (2002) arXiv:hep-th/0110101. 